\title{
Device-Free Home Intruder Detection and Alarm System Using Wi-Fi Channel State Information
}

\author{
Mohammed Abdulaziz Aide Al-qaness, Fangmin Li, Xiaolin Ma, and Guo Liu
}

\begin{abstract}
In this paper, we design a device-free intruder detection and alarm system, named WiGarde by exploiting off-the-shelf Wi-Fi channel state information (CSI) to detect an intruder through door or window. WiGarde extracts the CSI amplitude information across MIMO antennas. We implemented WiGarde with commercial IEEE 802.11 NICs and evaluated its performance in two cluttered indoor environments. The system is robust and avoids false alarm occurrence, owing to our novel bad stream elimination algorithm. To extract the best feature, we design a new method to intercept the segment of the signal of intrusion based on wavelet analysis and dynamic time window based on Short-time Energy. We adopt Support Vector Machine (SVM) algorithm to classify human intrusion; our SVM algorithm could classify intrusion process with general walking through the area of interest. We compare WiGarde with the previous approaches; results show that our system outperforms the corresponding best CSI-based and RSSI-based in both of static and motion states. Our system gained high accuracy of $94.5 \%$ in a dynamic environment for intrusion through door or window.
\end{abstract}

Index Terms-Intruder detection, device-free, CSI, home safety motion detection, WiFi.

\section{INTRODUCTION}

A huge of home monitoring and security systems had been invented, almost of the previous approaches exploited the combination of sensors and GSM network. WSN has the advantages of broad covering area. It can remotely monitor, and establishes a fast network. GSM has the advantages of long communication distance and wide covering area [1]. Zhang et al. [2] proposed an indoor security system based on a combination of sensor nodes and ZigBee connected with jumping robot. The sensor nodes are installed above doors and windows in the house to detect an intruder, then send a message to the robot the robot can jump to take a photo and send it to the gateway. Sensors based monitoring and alarm systems have been leveraged many technologies such as GSM-based [3], and 3G-based [4]. However, such systems require special installation with a high cost.

The wide spread of WLAN and its low cost has motivated researchers into device-free localization and motion detection.

Manuscript received July, 17, 2016; revised August 19, 2016. This work is supported by National Natural Science Foundation of China with Grant Nos.61373042 and 61502361.

Mohammed Abdulaziz Aide Al-qaness is with the School of Information Engineering, Wuhan University of Technology, Wuhan 430070, China (e-mail: alqaness@whut.edu.cn).

Fangmin Li is also now with Department of Mathematics and Computer Science, Changsha University, Changsha, 410022, China (e-mail: 1fm@ccsu.edu.cn).

Xiaolin Ma and Guo Liu are with the School of Information Engineering, Wuhan University of Technology, China.
Youssef et al. [5] introduced the concept of device-free passive DfP localization, which enables the detection and tracking of entities that do not carry any devices nor participate in the localization process. Kosba et al. [6] utilized the received signal strength (RSS) to capture the environmental changes, which fluctuate when an intruder enters an area of interests. RSS based device-free approaches have a limitation due to the variability of RSS caused by the environment changes; it caused a false alarm detection. The new trend metric in device-free motion detection based on channel state information (CSI), which attracts more attention during recent years, since CSI can be exported from commodity wireless NICs [7]. CSI is capable of detecting an anomaly that affected by environment changes. CSI reflects the varying multipath reflection caused by intruder's existence due to its frequency diversity [8]. Compare to RSSI, CSI is fine-grained channel information, whereas, RSSI is coarse-grained channel information. The main differences are in two aspects: (1) RSSI is an average value of the signals received. CSI contains more information about the fading channel through amplitude and phase. (2) RSSI is severely affected by multipath effects. By observed the variation of CSI caused by body movement found that: in a static environment, CSI will maintain its stability in time, and when movement occurs, CSI will present burst mode. Based on this phenomenon, Xiao et al. [9] presented a method of indoor fine-grained motion detection system based on the time stability and frequency diversity of OFDM physical layer CSI. Zhou et al. [10] modelled the CSI subcarrier amplitude as a histogram, and applying EMD algorithm for signal classification, then built a fingerprint database, and designed a passive omnidirectional human detection system Omni-PHD, which can effectively detect the full range of human emergence. Qian et al. [11] leveraged the amplitude and phase information of CSI and the spatial diversity provided by MIMO to improve the accuracy and robustness of human motion detection, then applied support vector machine method to determine human motion, and designed a passive detection of moving humans with dynamic Speed (PADS). In this paper, we will introduce a novel device-free intruder detection in a real implementation. Unlike previous approaches, we focus on door and window intrusion, and we discriminate the intrusion action with similar activities.

In this paper, we use Naïve Bayesian classifier to eliminate the "bad streams"; then, the system could choose the useful streams that can expose information of human motion and leverage such information to detect door or window intrusion, which make our system robust and avoided errors caused by using median stream [11]. We also design a novel dynamic time window based on Short-time Energy to accurately 
intercept signal segment of the duration of intrusion. This novel method enables us to extract more meaningful features from the time domain and frequency domain of CSI for our action classifier. Comparing walking inside a home with through door intrusion action, CSI is more sensitive to inside room walking activity, and intrusion is more affect CSI than walking aside the door; so that, we need a high accurately classification model, we build our classification method on the famous known Support Vector Machine.

Our main contributions are summarized as follows:

1) In this paper, we propose a novel scheme for device-free intruder detection in a real implementation. To the best of our knowledge, WiGarde is the first device-free approach focuses on door and window intrusion, which be an applicable system for home safety.

2) We design an efficient bad stream elimination algorithm which effectively eliminates useless streams that may occur because the surrounding electromagnetic noise.

3) We design a novel method to intercept the segment of the signal of intrusion based on wavelet analysis and dynamic time window based on Short-time Energy.

4) We adopt SVM classification algorithm to detect doors and windows intrusion.

The rest of this paper organized as follows. In Section II, we summarize the related works. In Section III, we describe the system architecture and implementations. The experiments evaluation is explained in Section IV, and Section V is the conclusion.

\section{RELATED WORK}

In this section, we summarize some of the related works. Related works could be categorized into device-based and device-free systems. Device-free approaches contain RSSI-based, RF-based (Wi-Fi Radar), and CSI-based.

\section{A. Device-Based Systems}

There are many systems rely on the combination of sensors and internet for home security, Zhang et al. [2] proposed an indoor security system based on a combination of sensor nodes with ZigBee connected with jumping robot. The sensor nodes are installed above the doors and windows at the house to detect an intruder, then send a message to the robot; the robot can jump to take a photo and send it to the gateway. El-Medany et al. In literature [3], the authors described the architecture and simulation of a GSM based remote sensing and control system using FPGA. Zhang et al. [4] introduced a home automation system using GSM, the Internet, and speech recognition. Kim et al. [12] designed a Wireless (Universal Serial Connector) USB- Based Home Security System on the Open Services Gateway Initiative (OSGi) Service Platform used with a USB-based wireless controller. Zeki et al. [13] presented infrared sensors based motion detection system, which captures the image of any intruding persons and shares it with the client in both Android platform and in an online portal display. However, several of device-based home security systems have been deployed such as a combination of ZigBee and GSM [14], and GSM/GPRS [15], but device-based systems still have many limitations due to the high cost and cumbersome deployment.

\section{B. Device-Free}

Many approaches have been presented by using Wifi for indoor localization and motion detection, but none of the previous works focused on door and window intrusion. Here, device-free related works summarized as follows.

\section{1) RSSI-based}

In the last decade, RSSI has attracted researchers to use this metric in localization, motion detection, and activity recognition. In the motion detection approaches [5], [6], [16]-[18] the authors leveraged received signal strength (RSS) to capture environmental changes, which become anomalous when an intruder enters an area of supervision. Moore et al., [16] presented a system positioned human motion using the change in standard deviation of the received signal strength between stationary transmitters and receivers at known locations. Sabek et al. [17] developed RSS-based Spot system for the purpose of multi-entity detection and tracking. Yang et al. [18] proposed a joint intrusion learning approach which detects different intrusion patterns at the same time. However, RSS is prone to be affected by narrowband interference and multipath interference and thus become unstable. Specifically, the high variability of RSS makes it less sensitive to environmental variance caused by human motion. Therefore, it can't precisely detect the motion of human, thus lead to a false detection alarm. From another aspect, all of these works didn't focus on the door and window intrusion.

\section{2) Software defined radio (SDR) based}

Recently, researchers have adopted Software defined radio (SDR) in human motion detection and activity recognition. Adib et al. [19], [20] presented WiTrack and WiTrack 2.0, a device-free system which can track human movements through walls. They used Frequency Modulated Carrier Wave (FMCW). However, such approaches need a special hardware such as Universal Software Radio Peripheral (USRP).

\section{3) CSI-based}

Recently various approaches have leveraged channel state information for indoor localization and activity recognition. Xiao et al. [21] presented a CSI-based human motion detection system (Pilot), which can effectively detect the presence and location of the entity. However, the system only uses the first antenna of IWL 5300 NIC. In CSI-MIMO fingerprint positioning system [22], the MIMO information and the CSI amplitude and phase information of each subcarrier are comprehensively utilized to obtain the position accuracy. Omni-PHDs [10], [23], study omnidirectional sensing coverage for passive human detection, using multipath effects captured by CSI. Xi et al. [24] presented a device-free crowd counting system (FCC). FCC observes the variation of CSI and its relationship with the number of moving people. Xiao et al. Xiao et al. [9] proposed a method of indoor fine-grained motion detection system based on the time stability and frequency diversity of OFDM physical layer CSI. This system realizes motion detection by using the normal mode (i.e., CSI mode) in the static environment, and proposes a maximum eigenvalue method to extract and characterize the static and dynamic signal mode, and then perform the detection of the moving behaviour by applying density-based spatial clustering with the noise (DBSCAN) 
algorithm. Qian et al. [11] leveraged both amplitude and phase information of CSI and the spatial diversity provided by MIMO and designed a passive detection of moving humans with dynamic speed (PADS). However, there are still some problems with these systems. All of these systems still suffer from bad streams occurrence which in general cause a false alarm. Moreover, all of these systems didn't specify door and window intrusion.

\section{SYSTEM ARCHITECTURE AND IMPLEMENTATION}

WiGarde consists of two parts AP (Access point with MIMO antennas) and DP (laptop With MIMO Antennas), and works in two phases (1) short offline phase (35 s) where no human presence in the test bed, (2) detection phase, where the system detects an intruder from the door or window. As shown in Fig. 1, WiGarde consists of the following modules: Data collection module, Bad stream elimination module, Features extraction module and classification module.

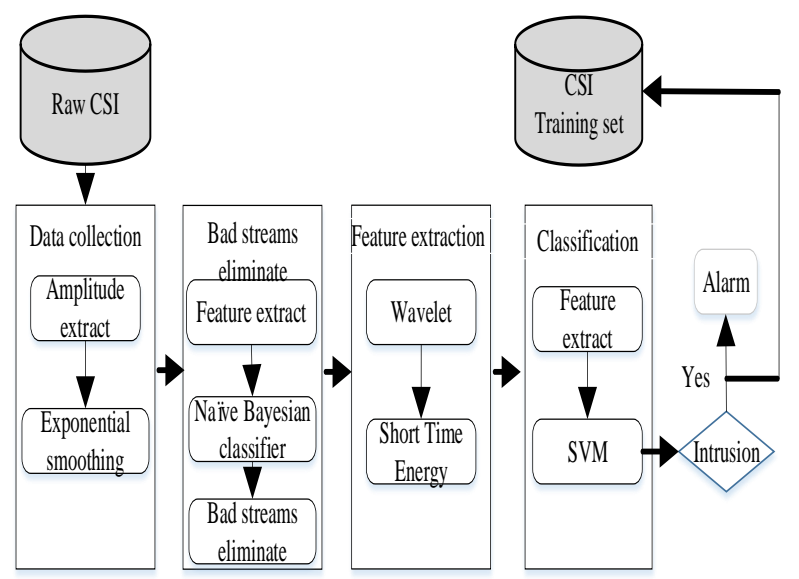

Fig. 1. System architecture and workflow.

\section{A. Data Collection Module}

We collected CSI data based on the open source CSI-tools [7]. We exploit $2 * 3$ MIMO technology, two antennas for transmitting (TP-LINK AP) and three antennas for receiving (Intel Wireless Link (IWL) 5300 card). Consequently, raw CSI can be divided into 6 streams; each stream has 30 subcarriers, thus, lead to get enough information to detect human intrusion motion, because each packet has 180 groups of CSI, as described in Equation (1):

$$
H=\left[\begin{array}{cccc}
h_{1,1} & h_{1,2} & \ldots & h_{1, n_{t}} \\
h_{2,1} & h_{2,2} & \ldots & h_{2, n_{t}} \\
\vdots & \vdots & \ddots & \vdots \\
h_{n_{r}, 1} & h_{n_{r}, 2} & \ldots & h_{n_{r}, n_{t}}
\end{array}\right] \in \boldsymbol{C}^{n_{r} \times n_{t}}
$$

each element in the matrix is complex represented as Equation (2) [25]:

$$
H_{i, j}=\left\|H_{i, j}\left(f_{k}\right)\right\| e^{\angle H_{i, j}\left(f_{k}\right)}
$$

where $f_{k}$ is the central frequency of the OFDM subcarrier that defined in 802.11n protocol, $H_{i, j}\left(f_{k}\right)$ is the amplitude, and $\angle$
$H_{i, j}\left(f_{k}\right)$ is the phase. We only use amplitude information of CSI as the indicator of human actions, since the phase of CSI is kind of random distribution in the time domain, which makes it hard to extract useful information.

After the CSI collection stage, the raw CSI suffer from the electromagnetic noise from the surrounding environments. The real trend of the signal is drowned in the noise. As usual, the fluctuations caused by human motions on CSI, mainly exist in the low frequency band, while the electromagnetic noise exists in the high-frequency band. Therefore, we design an exponential filter to filter the high frequency noise. As shown in Fig. 2(a,b) our filter eliminates noise effectively and does not distort trends caused by human motions.

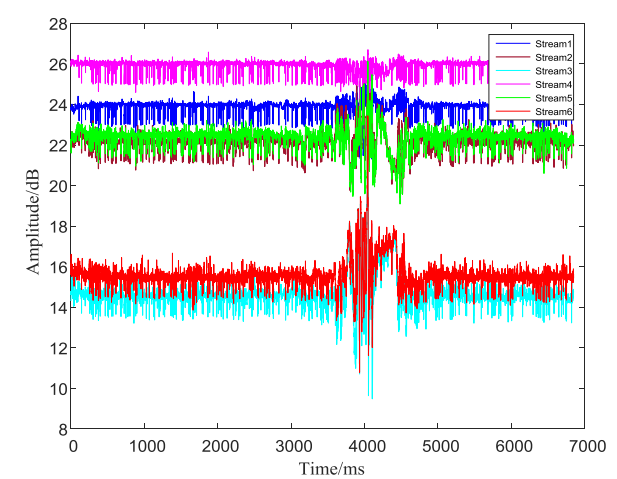

(a)

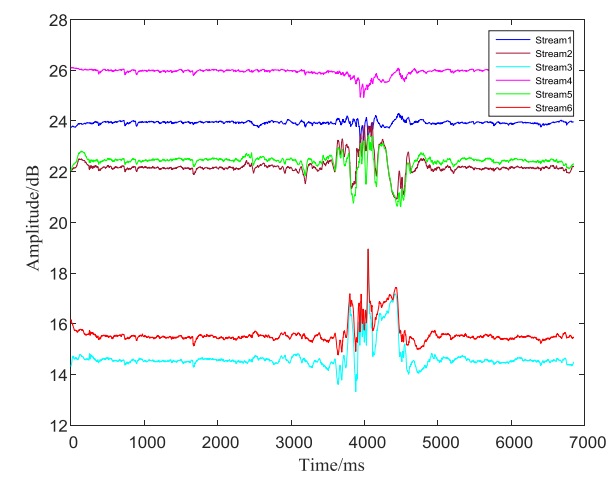

(b)

Fig. 2 (a) Raw CSI with noise; (b) Raw CSI after applying exponential filter.

\section{B. Bad Stream Elimination Module}

To avoid miss-detection or false alarm occurred by "Bad streams" which occurred in different antenna pairs, we use Naïve Bayesian Classification to eliminate bad antenna and extract the mean of "best ones" for accurately detect human intrusion. Our fast "bad streams" elimination algorithm calculates the following three features on every spatial diversity: (1) the difference between the maximum peaks and valleys (Max-Min), (2) mean value (Means) and (3) standard deviation (STD).

These three features are put into Naïve Bayesian classifier as a vector. Fig. 3 depicts the workflow of our fast bad streams elimination algorithm.

In case of bad streams occurrence, as shown in Fig. 2 (stream 1,4), the amplitude streams are almost flat, there are not any amplitude fluctuations, which lead to a false alarm; By applying our bad streams elimination algorithm, we can eliminate the bad streams and use only the best ones as shown in Fig. 2 (streams 2,3,5,6) the fluctuation of the CSI amplitude 
is very clear.

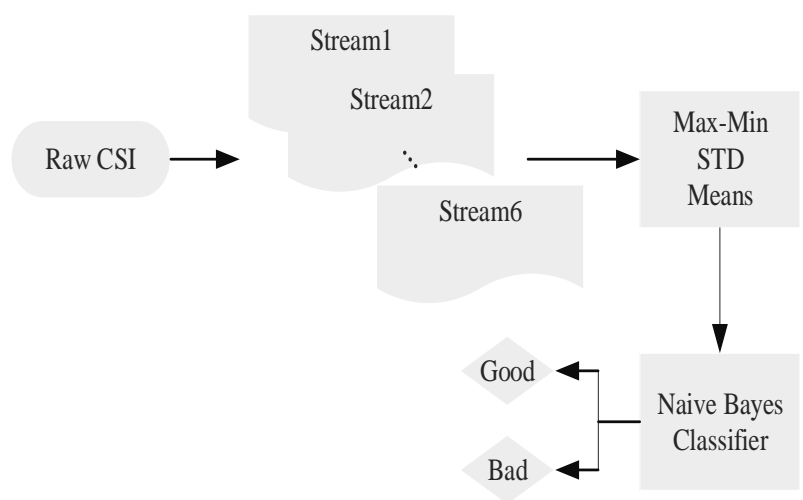

Fig. 3. Bad streams elimination algorithm workflow.

\section{Feature Selection Module}

We design a novel algorithm in order to get the width of the dynamic time window and the moment when intrusion emerges using wavelet. Wavelet algorithm lead to accurate extraction and analyzing of the CSI segmentation in the period of human motion, in further this algorithm is very necessary for accurate classification because as known the duration of human motion is a very important feature to distinguish different motions when we implement the classification algorithm. As shown in Fig. 4 from door or window intrusion action caused fluctuation in the raw CSI. It is very suitable to determine the time point when motion occurs.

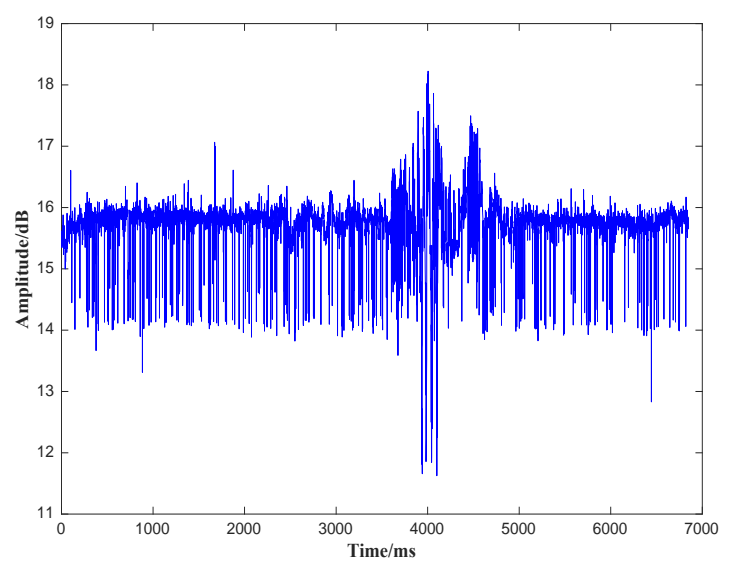

Fig. 4. Intrusion experiment raw CSI.

Fig. 5 shows the result we use wavelet analysis on the raw CSI. We can see that the first wavelet decomposition finds the time point accurately. Then, we use the short-time energy algorithm to discriminate intrusion and non-intrusion segments of raw CSI. As known, the short-time energy is the energy of short speech segment [26]. In Speech signal processing, short-time energy is a simple and effective classification algorithm which detects voiced and unvoiced segments [27]. Hereby we use the short-time energy as a metric to discriminate the normal state (non-motion) and abnormal state (motion). The variance of the raw CSI will increase with human activity, such walking, intruding through door or windows, or walking aside the door. Short-time energy could segment this abnormal signals, and then the different segmentations could be classified by SVM. As the same concept of the speech signal segmentation, the short time energy will increase with motion due to the variation of CSI, so the higher energy represents the motion state, and the lower energy means static state. We apply STE as the same description in our previous paper [28]. From Fig. 6, we see that energy of motion segment is higher than that of the non-motion signal of CSI signal. Experiments showed that it is an efficient metric of discriminating motion and static signals. Implementation and evaluation showed the validity of our segmentation and windowing method.

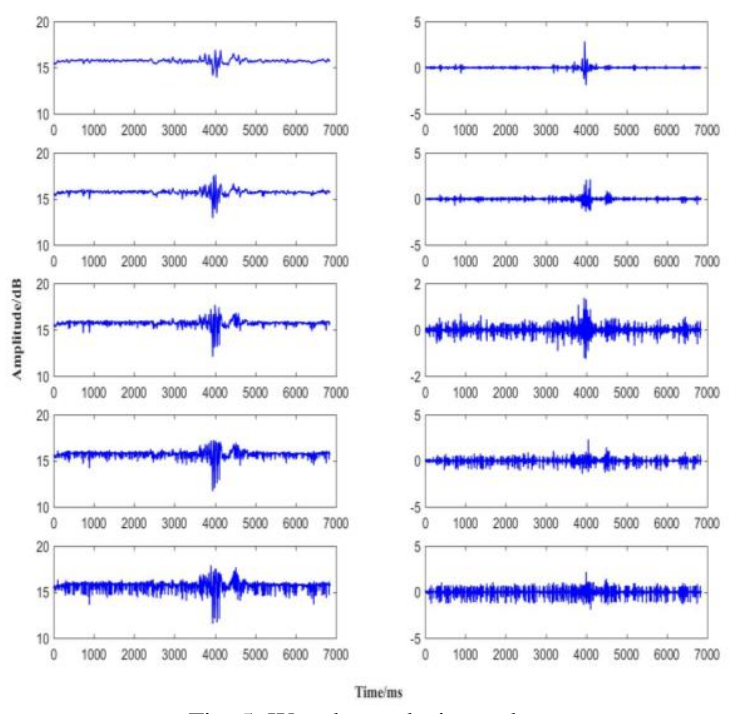

Fig. 5. Wavelet analysis results.

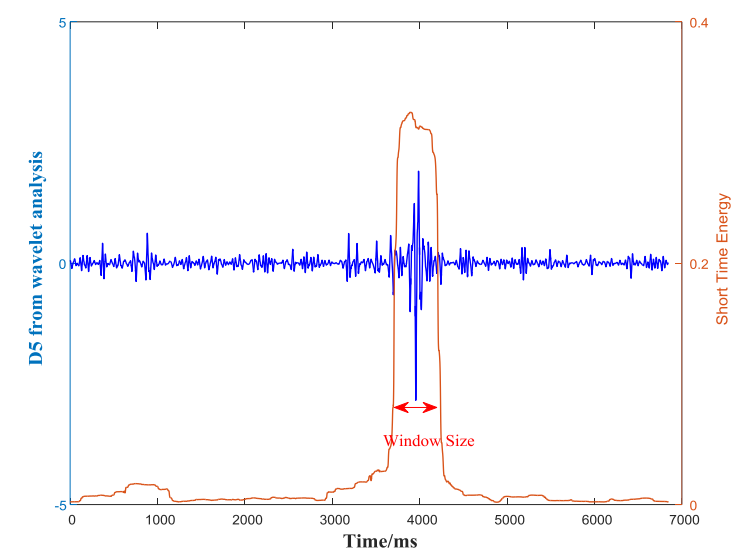

Fig. 6. Determine the width of the dynamic time window by short-time energy.

\section{Classification Module}

After detecting and segmenting the abnormal CSI signals, we need to classify these segmentations; we need to leverage the most correct classifying to avoid miss detection and false alarm. We applied several classification methods, then we adopt the most appropriate classification algorithm which is the Support Vector Machine (SVM). For getting a high accuracy, the safety system must classify the intrusion with another similar activity, for instance, walking on the corridor beside the door or standing beside door must differentiate with opening door, walking inside room also must be discriminate with intrusion action.

We apply one-class SVM classification algorithms to classify three motion activity, intrusion through doors or windows, walking slowly aside the door outside the room, and 
walking inside the room in NLOS place. We utilize the LIBSVM [29]. We choose five features to describe a human motion: (1) normalized standard deviation (STD); (2) median absolute deviation (MAD); (3) interquartile range (IR); (4) signal entropy; (5) duration of human motion.

\section{EXPERIMENTS AND EVALUATION}

\section{A. Test Bed and Equipment}

We evaluate WiGarde in two deferent environments, information Engineering Lab in JH Building of Wuhan University of technology, and Foreigner Student dormitory. We chose two different environments to test the generality of our system. Both environments have piled with various office facilities such as computers and desks (lab) and desks, beds, cupboard etc. (in a dorm), creating a rather complex multipath environment, as depicted in Figure 7(a,b). Figure $8 \mathrm{c}$ shows the test equipment which is an access point AP (TP-LINK TL-WR845N) with two antennas, and a detection point DP (ThinkPad x201) equipped with three antennas Intel Wi-Fi Link (IWL5300). During the experiments, we set the period of each experiment to $60 \mathrm{~s}$, and the motion begins at about $35 \mathrm{~s}$. The packet rate is $100 \mathrm{HZ}$ so that, we can get enough information for detecting human intrusion accurately.
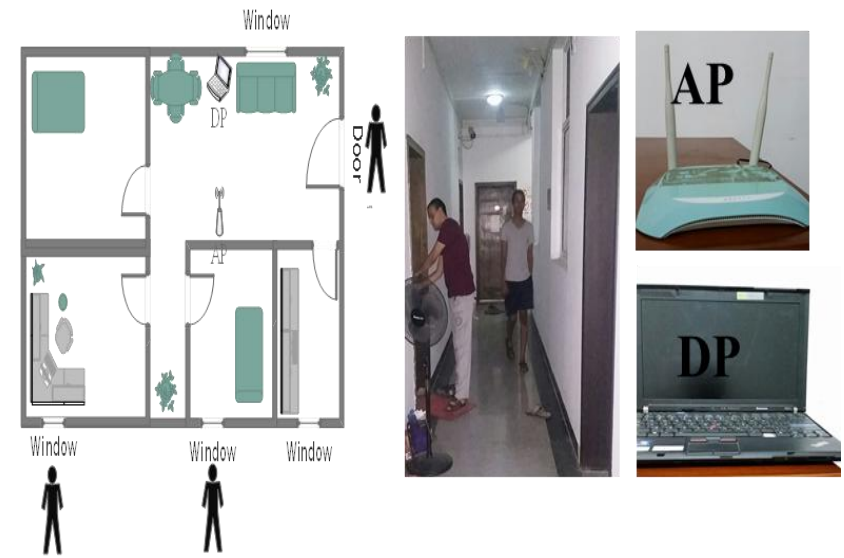

Fig. 7. Experiment test bed, and equipment.

\section{B. Results}

We used the same metrics mentioned in PADS [11] and we compare our system with PADS and RSSI-Based, the two metrics as following:

- True Negative (TN) rate: TN rate is the probability that the static environment is correctly classified.

- True Positive (TP) rate: TP rate is the probability that the human intrusion (through doors or windows). For true negative metric, we conduct many experiments in 5 different places: (1) Lab room (2) corridor, (3) classroom, (4) dorm room, (5) dorm hall.

As shown in Fig. 8, our system is more accurate and outperforms both systems in the static environment.

For intrusion through doors or windows detection, we conducted exhaustive experiments. A volunteer is asked to go through the corridor and open the door then closes the door and stay away without movement. Moreover, a volunteer is asked to open window from outside and stay without moving.
In each place, we collected 500 samples from 5 different volunteer users. We measure the $5^{\text {th }}$ cross validation of the collected samples of each user.

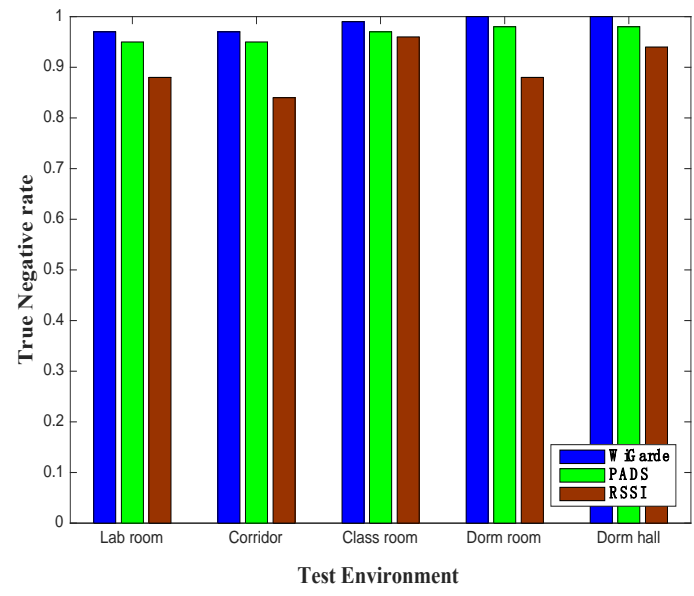

Fig. 8. TN rate in static case.

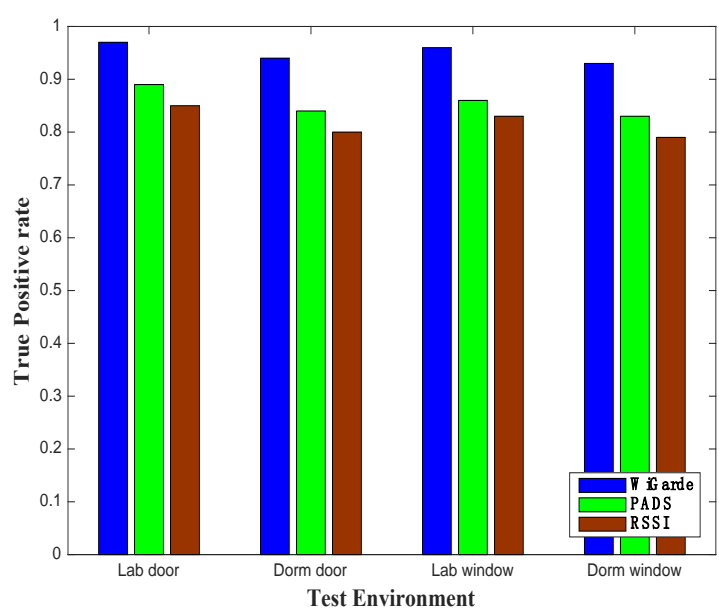

Fig. 9. TP rate for intrusion detection

We compare our system with PADS and RSSI based in four states: intrusion from lab door, dorm door, lab window, and dorm window. As shown from Fig. 9, our system outperforms PADS and RSSI-based. Our system has a positive rate for intrusion process of $94.5 \%$ that means its validity to save a home from outlier intrusion through both door and window.

Furthermore, to describe the bad effects of bad streams, we test the true positive rate with and without bad stream elimination algorithm. As shown in Fig. 10, the detection rate decreases to $84 \%$, which may lead to a false alarm.

The above-mentioned experiments as the same as most of the previous device-free human motion detection approaches, during experiments no another human inside test area. To study the effect of human interference, we conduct some experiments while other human presence in the test area in three scenarios:

1) Intrusion process while no human in the test area;

2) One human in the test area: we ask one volunteer to stroll inside the room while the other one is intruding through door or windows.

3) Tow human presence: We asked two volunteers to stroll inside the room while the intruder volunteer intruding through door or window.

As shown in Fig. 11, the presence of multiple moving objects inside room or house could decrease the accurate 
detection, the accuracy decreased to $83 \%$ and $69 \%$ for 1,2 humans, respectively.

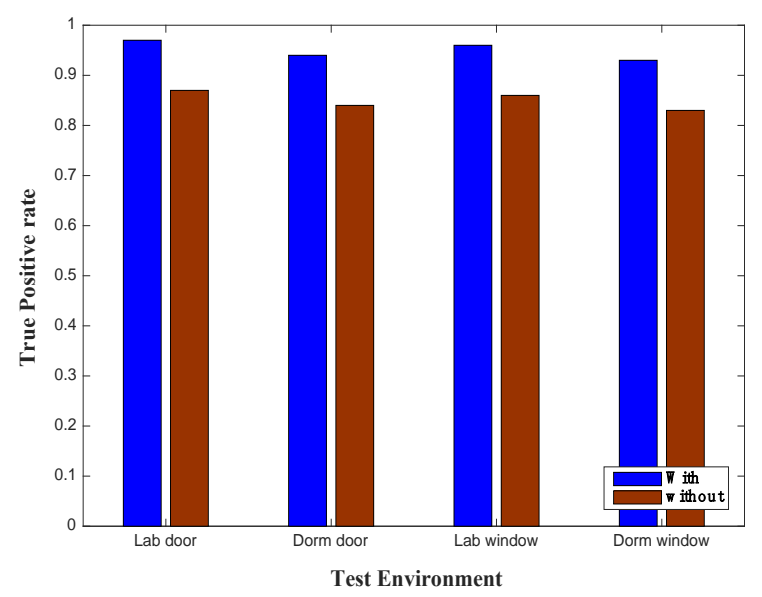

Fig. 10. TP rate with and without bad streams elimination.

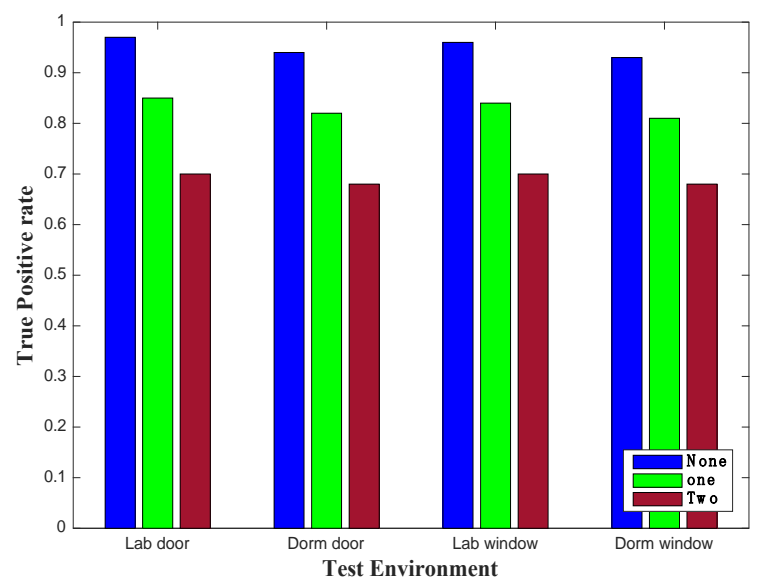

Fig. 11. TP rate with appearance of one or two human.

\section{CONCLUSION}

In this paper, we present WiGarde, a device-free intruder detection, based on channel state information of Wi-Fi signals. Our system detects human intrusion through doors or windows in dynamic environments with high and stable accuracy. We compare WiGarde with previous approaches and find that it outperforms previous approaches in human detection accuracy. At the same time, WiGarde is the first approach dedicated in doors and windows intrusion process. Further, the proposed system is robust and applicable to the real-world dynamic environment, due to the bad stream elimination algorithm, which avoids the bad streams occurrences raised by interference signals.

We have conducted more than 200 experiments in different dynamic environments. The evaluation results show that the system is applicable and can be improved further to get the complete house safety system. We have gained accuracy of $94.5 \%$ for doors and windows intrusion while no one moves inside a room, whereas the false alarm is about $1.7 \%$. The accuracy decreases to $84 \%$ in case of one human move inside a room, and $69 \%$ while two humans move inside a home. These results imply that the appearance of multiple people could decrease the accuracy of WiGarde which implies that it is valuable to improve the proposed system and develop new whole-home safety system in the future work.

\section{REFERENCES}

[1] H. Huang, S. Xiao, X. Meng, and Y. Xiong, "A remote home security system based on wireless sensor network and GSM technology," in Proc. the Second International Conference on Networks Security, Wireless Communications and Trusted Computing, Wuhan, Hubei, China, 24-25 April 2010, pp. 535-538.

[2] J. Zhang, G. Song, G. Qiao, T. Meng, and H. Sun, “An indoor security system with a jumping robot as the surveillance terminal," IEEE Transactions on Consumer Electronics, vol: 57, no. 4, pp. 1774-1781, November 2011.

[3] W. M. El-Medany and M.R. El-Sabry, "GSM-based remote sensing and control system using FPGA," in Proc. the International Conference on Computer and Communication Engineering (ICCCE), Kuala Lumpur, Malaysia, 13-15 May 2008, pp. 1093-1097.

[4] Y. Zhang, G. Zhao, and Y. Zhang, "A smart home security system based on 3G," in Proc. the International Forum on Computer Science-Technology and Applications, Chongqing, China, Dec. 2009, pp. 291-294.

[5] M. Youssef, M. Mah, and A. Agrawala, "Challenges: Device-free passive localization for wireless environments," in Proc. ACM MobiCom07, New York, USA 2007, pp. 222-229.

[6] A. E. Kosba, A. Saeed, and M. Youssef, "Rasid: A robust wlan device-free passive motion detection system," in Proc. IEEE PerCom, Lugano, Switzerland, 19-23 March 2012, pp. 180-189.

[7] D. Halperin, W. Hu, A. Sheth, and D. Wetherall, "Tool release: Gathering $802.11 \mathrm{n}$ traces with channel state information," ACM SIGCOM Computer Communication Review, vol. 41, no. 1, pp. 53-53, Jan. 2011.

[8] A. Bhartia, Y. C. Chen, S. Rallapalli, and L. Qiu, "Harnessing frequency diversity in Wi-Fi networks," in Proc. the 17th Annual International Conference on Mobile Computing and Networking, ACM, 2011, pp. 253-264.

[9] J. Xiao, K. Wu, Y. Yi, L. Wang, and L. M. Ni, "FIMD: Fine-grained device-free motion detection," in Proc. IEEE ICPADS'12, Singapore, 17-19 Dec. 2012, pp. 29-235.

[10] Z. Zhou, Z. Yang, C. Wu, L. Shangguan, and Y Liu, "Omnidirectional coverage for device-free passive human detection," IEEE Transactions on Parallel and Distributed Systems, vol. 25, no. 7, pp. 1819-1829, July, 2014.

[11] K. Qian, C. Wu, Z. Yang, Y. Liu, and Z. Zhou, "PADS: Passive detection of moving targets with dynamic speed using PHY layer information," in Proc. IEEE ICPADS'14, Hsinchu, Taiwan, 16-19 Dec. 2014, pp. 1-8.

[12] H. S. Kim and C. G. Lee, "Wireless USB-based home security system on the OSGi service platform," in Proc. International Conference of Consumer Electronics (ICCE 2007), Las Vegas, USA, 10-14 Jan. 2007, pp. 1-2.

[13] A. M. Zeki, E. E. Elnour, A. A. Ibrahim, C. Haruna, and S. Abdulkareem, "Automatic interactive security monitoring system," in Proc. International Conference on Research and Innovation in Information Systems (ICRIIS), Kuala Lumpur, Malaysia, 27-28 Nov. 2013, pp. $215-220$.

[14] A. W. Ahmad, N. Jan, S. Iqbal, and C. Lee, "Implementation of ZigBee-GSM based home security monitoring and remote control system," in Proc. 54th International Midwest Symposium Conference on Circuits and Systems (MWSCAS), Seoul, South Korea 7-10 Aug. 2011, IEEE, pp.1-4.

[15] Y. Zhao and Z. Ye, "A low cost GSM/GPRS based wireless home security system," IEEE Transactions on Consumer Electronics, vol. 54, issue 2, pp. 567-572, July 2008.

[16] R. S. Moore, R. Howard, P. Kuksa, and P. R.Martin, "A geometric approach to device-free motion localization using signal strength," Rutgers University, Technical Report DCS-TR-674, September, 2010.

[17] I. Sabek, and M. Youssef, "Spot demo: Multi-entity device-free wlan localization," in Proc. the 7th ACM International Workshop on Wireless Network Testbeds, Experimental Evaluation and Characterization, ACM, 2012, pp. 87-88.

[18] J. Yang, Y. Ge, H. Xiong, Y. Chen, and H. Liu, "Performing joint learning for passive intrusion detection in pervasive wireless environments," in Proc. INFOCOM IEEE, March 14-19, 2010, pp. 1-9.

[19] F. Adib, Z. Kabelac, D. Katabi, and R. C. Miller, "3D tracking via body radio reflections," in Proc. the 11th USENIX Conference on Networked Systems Design and Implementation, 2014, ACM, pp. 317-329.

[20] F. Adib, Z. Kabelac, and D. Katabi, "Multi-person motion tracking via RF body reflections," in Proc. the 12th USENIX Conference on 
Networked Systems Design and Implementation, May, 2015, ACM, pp. 279-292.

[21] J. Xiao, K. Wu, Y. Yi, L. Wang, and L. M. Ni, "Pilot: Passive device-free indoor localization using channel state information," in Proc. 33rd International Conference on Distributed Computing Systems (ICDCS), Philadelphia, PA, USA, 8-11 Jul. 2013, pp. 236-245.

[22] Y. Chapre, A. Ignjatovic, A. Seneviratne, and S. Jha, "CSI-MIMO: Indoor Wi-Fi fingerprinting system," in Proc. IEEE39th Conference on Local Computer Networks (LCN), Edmonton, Canada, Sept. 8-11, 2014, pp. 202-209.

[23] Z. Zhou, Z. Yang, C. Wu, L. Shangguan, and Y. Liu, "Towards omnidirectional passive human detection," in Proc. IEEE INFOCOM'13, Turin, Italy, 14-19 Apr. 2013, pp. 3057-3065.

[24] W. Xi, J. Zhao, X.Y Li, K. Zhao, S. Tang, X. Liu, and Z. Jiang, "Electronic frog eye: Counting crowd using wifi," in Proc. IEEE INFOCOM, Toronto,Canada, April 27 2014-May 2 2014, pp. 361-369.

[25] A. Goldsmith, Wireless Communications, Cambridge University Press, UK, 2005.

[26] X. Yang, B. Tan, J. Ding, J. Zhang, and J. Gong, "Comparative study on voice activity detection algorithm," in Proc. International Conference of Electrical and Control Engineering, ICECE, Wuhan, China, June 25-27, 2010, pp. 599-602.

[27] D. Enqing, L. Guizhong, Z. Yatong, and C. Yu, "Voice activity detection based on short-time energy and noise spectrum adaptation," in Proc. 6th International Conference on Signal Processing, Aug. 26-30, 2002, pp. 464-467.

[28] M. A. A. Al-qaness and F. Li, "WiGeR: WiFi-based gesture recognition system," ISPRS International Journal of Geo-information, 2016, vol. 5, no. 6, p. 92, June 2016.

[29] C. C. Chang and C.J. Lin, "LIBSVM: A library for support vector machines," ACM Transactions on Intelligent Systems and Technology (TIST), 2011, vol. 2, no. 27, pp. 1-27.

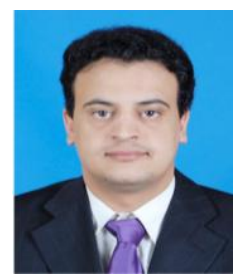

Mohammed Abdulaziz Aide Al-qaness is a research scholar in the Department of Information Engineering, Wuhan University of Technology, Wuhan, China. He received his master in information and communication engineering from Wuhan University of Technology, Wuhan, China in 2014. He has published several papers in mobile computing and indoor human tracking \& activity recognition. His research interests include wireless sensing, mobile system and mobile computing.

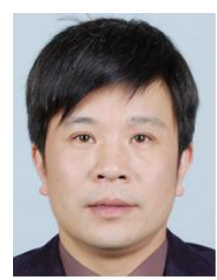

Fangmin $\mathbf{L i}$ is a professor at the School of Information Engineering, Wuhan University of Technology. He received his BS from the Huazhong University of Science and Technology, Wuhan, China, in 1990, MS from the National University of Defence Technology, Changsha, China, in 1997 and the PhD from Zhejiang University, Hangzhou, China, in 2001, all in computer science. He has authored several books and over 30 academic papers in wireless networks, and also holds tens of Chinese patents. His current research interests include wireless communications and networks, computer systems and architectures.

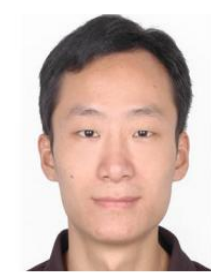

Xiaolin Ma is a lecturer at the School of Information Engineering, Wuhan University of Technology. He received the $\mathrm{BS}, \mathrm{MS}$ and $\mathrm{PhD}$ degrees from Wuhan University of Technology in 2008, 2012 and 2014, respectively, all in information and communication engineering. He has published tens of papers in wireless networks and holds one Chinese patent. His current research interests include wireless sensing, mobile computing and machine learning.

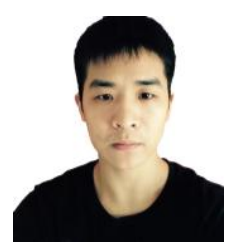

Guo Liu is a master student at the School of Information Engineering, Wuhan University of Technology. He received his BS degree from Wuhan University of Technology in 2014 in communication engineering. He has published several papers in mobile computing and indoor localization. His current research interests include wireless sensing, mobile system and mobile computing. 